Association by Unconscious Middle Factors. Jerusalem, Wundt.-Phil. Stud., x., ii.

Dr. Jerusalem communicates an experience of Herr Baumgarten, purporting to show that the idea or memory-image of two people, vividly called up by the unnoticed scent of a flower (which was not detected till after the memory-image had arisen in consciousness) implies that " the smell-sensation was here a really unconscious middle factor." $\mathrm{He}$ adduces this in support of Scripture's views (Phil. Stud., vii.).

Wundt rejoins that the scent of the flower was " unrecognised" (unbemerkt), not unconscious (unbewusst). Baumgarten's observation is interesting, because the unrecognised middle factor is here present in consciousness, whereas in Scripture's experiments the middle factors were not direct sensations during the process of mediate association, but were reproduced (vii., p. 78). Here the scent was perceived, though not apperceived, i.e., not distinguished from the scent of the other flowers. Wundt, therefore, calls this " a case of mediate association through middle factors present in consciousness, but only dimly perceived there."

Frances A. Welby.

\title{
Dana and Ketscher on Paralysis Agitans.
}

I.-In some preliminary remarks on the clinical phenomena of the disease, Dana (New York Medical Journal, June 10, 1893), emphasizes three classes of symptoms.

(1) Motor symptoms, chiefly due to difficulties in commencement of movements, or, as the author terms it, "a hitch in volition."

(2) Vasomotor symptoms, flushings, sweats, subjective sensation of heat, peculiarities of pulse. He believes there is a general vasomotor paralysis, affecting the vessels of the cord and the nerve trunks, in addition to those of the skin.

(3) Disturbances of the blood, and of metabolism. Purpura hæmorrhagica occurs sometimes, glycosuria sometimes; and in the opinion of some authors, phosphaturia. The author examined the blood in two cases; in one, the chief abnormality was absence of eosino-philous cells; in the other, there was " anæmia of chlorotic type; lessened percentage of hæmoglobin, and decreased resistance of red cells." 
(4) Sensory symptoms, viz., sciatica, neuralgia, and rheumatic pains; distressing sensations of heat and cold.

He then turns to the pathology of the disease with which his paper is chiefly occupied. He quotes 44 cases of paralysis agitans, real or supposed, in which post-mortems have been made, but out of these he rejects all but fourteen, as being in his opinion scientifically valueless. Upon the cases of Dubief, von Sass, Koller, and Borgherini, he places most stress. In the bulk of these cases certain anatomical facts appear, which although rather vague may possibly be taken as the basis of the morbid anatomy of this disease. They are degeneration of the vessels, enlargement of the perivascular spaces, proliferation of the neuroglia and connective tissue in the neighbourhood of the vessels; pigmentation and some degeneration of the spinal motor cells, and of those in the bulbar nuclei ; thickening of the pia mater surrounding the cord, with sclerosis of the neighbour. ing periphery of the cord; blocking of the central spinal canal by proliferation of cells; changes in the cells of the sympathetic.

The author's own case is as follows:-A woman, age about 58 at time of death, died from exhaustion five years after onset of the disease. The symptoms began after a fright, and consisted of pain and weakness in the back; tremors of the limbs, followed by weakness; stooping gait; slowness of movement, \&c. The brain and cord were hardened in Müller's fluid. In the cortex cerebri was some increased vascularity, dilatation of the vascular spaces; and some trivial changes in the nerve cells.

In the nuclei of the seventh, ninth, tenth, and eleventh cranial nerves was pigmentation, with some degeneration of the cells.

In the cord, the pia mater, particularly of the cervical and lumbar regions, was thickened; while in the lateral columns beneath was some diffuse infiltration of connective tissue, which had not however eaused much degeneration of the nerve fibres. The cerebellar tracts were not affected by this sclerosis. The vessels in the anterior cornua were much dilated, particularly the veins. The anterior cornual cells were somewhat shrunken and pigmented, and took stains irregularly. The central canal, at some levels, was filled with débris and exsudate. The anterior nerve roots were congested, and perforated with dilated vessels.

In a second case, the details of which are not fully given, the author found marked thickening of the spinal pia mater; with diffuse interstitial sclerosis, of the lateral columns especially; congestion of the central and anterior gray matter; and slight changes in the nerve cells. 
The anatomical seat of the disease the author places in the spinal cord, medulla and pons, and more particularly in the blood vessels of the central and anterior gray matter of the cord, and in the lateral columns (pyramidal tracts, lateral fundamental columns, and lateral limiting layers). As to the nature of the anatomical change we quote his own words:- "This anatomical basis I believe to be essentially as follows: First, a congestion of the central and anterior parts of the gray matter of the cord with some thickening of the blood vessels, but not very much, and some changes of the external wall of the nature of periarteritis. With this congestion and vascular irritation there occurs a proliferation of connective tissue, which especially invades the lateral columns of the spinal cord. In severe cases it affects also the periphery, producing a leptomeningitis and a zone of sclerosis surrounding the cord in a variable degree. The congestion with dilatation of blood vessels affects especially also the anterior roots, to a less extent the posterior roots. There is degeneration of the cells of the anterior horns, which, however, occurs only late in the disease and does not affect all the cell groups. The central cells or the cells of the median and central areas, which we suppose to be more connected with vasomotor and glandular functions, seem to be especially involved. There is also-and this is an important fact to bear in mind-a decided loss of the fibre net-work in the anterior horns and the central gray matter. The changes appear to involve especially the cervical and lumbar regions. Similar changes occur in the medulla and pons, but here they are less marked. Degenerative changes, however, are to be found, in many cases at least, in the cells of the nuclei of the glosso-pharyngeal and pneumo-gastric nerves. Such changes would explain the disturbances in the vasomotor function and heart action found in the disease."

He makes this further suggestion as to the causation of the disease, viz., that it is due to the presence, in the circulation, of some irritative toxine, which in its turn may originate in some glandular defect-the process being analogous to that of myxœdema. For this hypothesis, however, no evidence is adduced.

II.-Ketscher's paper (Zeitschrift für Heilkunde, 1892, p. 445) opens with a very complete account of the work already done upon the morbid anatomy of paralysis agitans. It would appear that, after dismissing those cases which probably were not genuine paralysis agitans at all, the remainder consist partly of cases in which no morbid appearances were discovered post- 
mortem (some of which, however, were not examined microscopically), and partly of others in which more or less definite results were obtained. These results may be summarised as-hyperplasia of the connective tissue and of the neuroglia in the spinal cord; changes of various degrees in the vessels, pigmentation of the ganglion cells, blocking of the central canal of the spinal cord, presence of corpora amylacea in more or less excess. Similar alterations have also been found in the brain, the peripheral nerves and muscles, and in the sympathetic system. Most investigators consider that the chief seat of the disease is in the spinal cord; though they are by no means unanimous on this point; and some distinguished men (Charcot, Oppenheim, \&c.) maintain that paralysis agitans is a "pure neurosis."

Ketscher investigated microscopically three cases of paralysis agitans; the ages of the patients were respectively 70,68 and 76. In all three cases he found changes in the central nervous system (mainly, it would appear, in the cord) ; in two cases changes in the muscles, in one case in the peripheral nerves. These changes he summarized as follows:-

(1) As to the parenchyma.

Scattered indication of degeneration and atrophy, viz., in the ganglion cells of brain and cord-pigmentation, indistinctness of outline, absence of nuclei, finely granular degeneration; in the nerve fibres (particularly of the posterior columns of the cord)-changes ranging from mere swelling of the axis-cylinder to complete disappearance of the nerve fibres; in the muscles-multiplication of nuclei, loss of transverse striation, byaline and fatty degeneration, disappearance of muscle-substance.

(2) As to the interstitial tissues.

There was general increase of the interstitial connective tissue. Around the cord the peripheral connective tissue was thickened, and broad bands extended from it towards the central parts, particularly in the posterior columns. In the peripheral nerves, the peri-neurium was thickened, and in some places the endo-neurium also. Similarly the connective tissue between the muscular fibres was thickened.

The neuroglia both of brain and cord was increased. The neuroglia layer of the cerebral cortex, and the ependyma of the ventricles was thickened. So was the glia of the cord, both the peripheral layer and that of the internal parts, and this overgrowth appeared to start from the neighbourhood of the vessels. In the posterior columns particularly this overgrowth penetrated 
not only between groups of fibres, which were thus marked off into separate bundles, but also between the individual nerve fibres in some places. The disease reached its maximum in the lumbar region of the cord. There were numerous corpora amylacea, in the ependyma of the lateral ventricles, in the superficial parts of the brain, in the peripheral parts of the cord, and along the posterior fissure of the cord.

The central canal of the cord was blocked with proliferated epithelial cells.

(3) As to the vascular system.

The vascular walls were thickened. Here and there were miliary aneurysms, or even small extravasations of blood. The adventitia of the cerebral vessels contained a good deal of pigment. The lymphatic spaces-peri-vascular and peri-cellular -were dilated, and contained sometimes coagulated lymph, sometimes leucocytes, sometimes blood. Here and there in the neighbourhood of these spaces an " cdematous softening" of the brain substance could be made out.

These changes, which have been observed by others besides himself, the author believes to be no fortuitous occurrence, but to stand in some causal relation to the disease.

A certain reservation has, however, to be made in view of the author's investigations into the condition of the nervous system in aged people generally. He made microscopical examination of the nervous system in ten aged people, dying from various causes; and comes to the conclusion that in old age changes are found resembling in kind those found in paralysis agitans, and differing only in their less advanced degree. He finally expresses bis agreement with the view of Jacobsohn, Dubief and Borgherini, that paralysis agitans is nothing else than senility of the nervous system, early in occurrence and intense in degree.

J. A. OrJierod, M.D. 